\title{
Platelets Kill Intraerythrocytic Malarial Parasites and Mediate Survival to Infection.-
}

Brendan J. McMorran, ${ }^{1}$ * Vikki M. Marshall, ${ }^{2 \# \dagger}$ Carolyn de Graaf, ${ }^{2,}{ }^{3}$ Karen E. Drysdale, ${ }^{1}$ Meriam Shabbar, ${ }^{1}$ Gordon K. Smyth, ${ }^{2}$ Jason E. Corbin, ${ }^{2}$ Warren S. Alexander, ${ }^{2}$ Simon J. Foote ${ }^{1}$

${ }^{1}$ The Menzies Research Institute, University of Tasmania, Private Bag 23, Hobart, Tasmania 7000, Australia. ${ }^{2}$ The Walter and Eliza Hall Institute of Medical Research, $1 \mathrm{G}$ Royal Pde, Parkville, Victoria 3050, Australia. ${ }^{3}$ Department of Medical Biology, University of Melbourne, Parkville, Victoria 3010, Australia.

*To whom correspondence should be addressed. E-mail:

brendan.mcmorran@utas.edu.au

$\dagger$ Present address: Peter MacCallum Cancer Centre Research Division, Locked Bag 1, A'Beckett Street, Victoria 8006, Australia.

Author: Inserted text appears in bold blue type; deletions are in red with strike-through marks. Please read the galley proofs and editorial changes carefully, check figures closely, and make any changes directly on the galleys.

Harry Jach, copyediting; tel: 202-326-6512; fax: 202-789-4669; e-mail: hjach@aaas.org 
Platelets play a critical role in the pathogenesis of malarial infections by encouraging the sequestration of infected red blood cells within the cerebral vasculature. But platelets also have well-established roles in innate protection against microbial infections. We found that purified human platelets killed Plasmodium falciparum parasites cultured in red blood cells. Inhibition of platelet function by aspirin and other platelet inhibitors abrogated the lethal effect human platelets exert on P. falciparum parasites. Likewise, platelet-deficient and aspirin-treated mice were more susceptible to death during erythrocytic infection with Plasmodium chabaudi. Both mouse and human platelets bind malarial-infected red cells and kill the parasite within. These results indicate a protective function for platelets in the early stages of erythrocytic infection distinct from their role in cerebral malaria. 
Adaptive immunity to malarial infection accumulates slowly over the lifetime of an individual living in an endemic region. Cross-_immunity between isolates is low, and every new infection requires the development of a virtually novel immune response. Therefore, innate mechanisms that limit parasite growth within the red blood cell (RBC) are extremely important in determining survival, especially during the first few years of life, because clinical severity correlates closely with parasite mass. Several known mutations affect the RBC and decrease the severity of infection through either impediment of parasite entry or development within the cell (1). However, we know little about other non-RBC mechanisms that may influence the course of infection before the onset of effective adaptive immune responses.

Thrombocytopenia is a common clinical accompaniment of Plasmodium falciparum, (2-4), Plasmodium vivax $(5,6)$, and Plasmodium chabaudi (7) malarial infections. Low platelet concentrations correlate with increased parasite density $(8,9)$ and poor outcome (10). Platelets bind preferentially to infected RBCs $(11,12)$ and have been postulated to play a role in the pathogenesis of malarial infection, either positively $(13,14)$ or negatively $(15,16)$. Given the role platelets play in other infectious diseases (17), we explored their influence on the course of a malarial infection in the mouse model, $P$. chabaudi, and in vitro using the cultured human malarial parasite, $P$. falciparum.

The megakaryocyte growth and differentiation factor, C-mpl, is the receptor for thrombopoietin and is encoded by the $\mathrm{Mpl}$ gene. Homozygous disruption of the Mpl gene on a C57BL/6 background $\left(\mathrm{Mpl}^{-/}\right)$results in mice with only one-tenth as many circulating platelets as their undisrupted counterparts have (18). Platelet-deficient $\mathrm{Mpl}^{-/-}$mice were significantly more susceptible to death from $P$. chabaudi than were isogenic C57BL/6 mice $(P<0.0001$, Mantel-Cox log-rank test on survival data), irrespective of well-known sex differences. In females, $5 \%$ of C57BL $/ 6$ versus $50 \%$ of $\mathrm{Mpl}^{-/-}$mice died. Twenty percent of the male C57BL/6 mice and all the male $\mathrm{Mpl}^{-/-}$mice died (Fig. 1, A and B).

Cohort analysis of groups of mice showed that platelet numbers decreased concomitantly with the appearance of parasites in the peripheral circulation (Fig. 1, E and F). Platelet numbers dropped to 10 te $20 \%$ of normal values in the C57BL/6 mice, with a smaller percentage drop in the $\mathrm{Mpl}^{-/}$mice. Platelet numbers reached a nadir before the onset of severe disease symptoms and anemia. No evidence of excess bleeding was seen in the $\mathrm{Mpl}^{-/}$mice during autopsy. Similar erythropoietic kinetics occurred between $\mathrm{Mpl}^{-/-}$and $\mathrm{C} 57 \mathrm{BL} / 6$ mice during both malarial challenge and experimentally-induced hemolysis (figs. S1 and S2), excluding differential erythropoiesis as a cause for the different susceptibilities. Nor were differences in immunological responses, including production of lymphocytes and inflammatory cytokines, observed between the mouse strains during infection (fig. S3).

Acetyl salicylic acid (aspirin) inhibits cyclooxygenases I and II, resulting in a decrease in thromboxane A2 and decreased platelet activation as seen by lower platelet aggregation (19). Mice were given $25 \mathrm{mg}$ of aspirin per kilogram of body weight intraperitoneally daily from the day before $P$. chabaudi infection until day 10 of infection and monitored for survival. C57BL/6 and $\mathrm{Mpl}^{-/-}$mice of both sexes treated withaspirin were significantly more susceptible than placebo- treated-mice (Fig. 1, C and D) (Cox proportional hazard analysis, $P=0.0009$ ). Thus, pharmacological inhibition of platelet function increases susceptibility to malarial infection in mice, although other indirect effects of aspirin cannot be excluded. 
Synchronized cultures of $P$. falciparum trophozoites were grown in human RBCs and incubated with increasing concentrations of purified human platelets. A concentration-dependent increase in the inhibition of parasite growth was observed (Fig. $2 \mathrm{~A}$ ). Growth inhibition (measured after 44 hours of incubation) peaked at $65 \%$ with $25-$ to 75 million platelets $/ \mathrm{ml}$. Most viable parasites observed at this time were mature trophozoites and shizonts. Reductions in mature shizonts at 5 hours and new rings at 21to 29 hours (fig. S4) suggest that platelets were inhibiting developmental maturation of the parasites.

Using the $P$. falciparum model, we investigated the dependence of parasite growth inhibition on platelet function by first incubating platelets with specific platelet antagonists. Maintenance of elevated intracellular adenosine 3',5'-monophosphate and guanosine $3^{\prime}, 5^{\prime}$ - monophosphate concentrations are key mechanisms in suppressing platelet activation $(20,21)$. Addition of the adenylyl cyclase activator prostaglandin E1 completely abrogated the platelet growth- inhibitory activity. Exposure of platelets to the guanylyl cyclase activator, NO (donated by sodium nitroprusside), also suppressed platelet-mediated effects on parasite growth (Fig. 2B). To test for the requirement of adenosine 5 '-diphosphate (ADP), an important physiological regulator of platelet activation [reviewed in (20)], we incubated the platelet- $P$. falciparum cultures with adenosine diphosphatase (ADPase) (22) to remove extracellular ADP from the culture medium. Unimpeded parasite growth in the presence of platelets and ADPase was observed. ADP-dependent platelet activation is mediated via two metabotrophic puronergic receptors, $\mathrm{P} 2 \mathrm{Y}_{1}$ and $\mathrm{P} 2 \mathrm{Y}_{12}$. Antagonism of $\mathrm{P} 2 \mathrm{Y}_{1}$, but not $\mathrm{P} 2 \mathrm{Y}_{12}$, abolished the parasite killing activity (Fig. 2B). Thus, $P$. falciparum growth is inhibited by platelets in a concentration-dependent and activation-dependent manner that requires ADP and activation through the $\mathrm{P} 2 \mathrm{Y}_{1}$ receptor.

As with $P$. chabaudi in the mouse, inhibition of cyclooxygenase by aspirin also prevented the inhibition of parasite growth by platelets (Fig. 2B). To approximate a clinical effect, a volunteer platelet-donor took aspirin orally twice daily $(4.2 \mathrm{mg} / \mathrm{kg})$ for a week before donating blood for platelet purification. These platelets were unable to inhibit growth of $P$. falciparum parasites (Fig. 2C), implying that aspirin is potentially harmful in malarial infections.

In mice infected with $P$. chabaudi for 8 days, with parasitemias at $\sim 10$ to $15 \%$, platelets were preferentially bound to infected RBCs. As shown with Giemsa-stained thin smears and light microscopy (Figs. 3A, i and ii), the percentage parasitemia was twice as high when only platelet-bound RBCs were counted compared with all RBCs (Fig. 3B). CD41 (a platelet surface antigen) on the surface of RBCs served as a marker for platelet binding. Three times as many infected RBCs stained for CD41 as did uninfected RBCs (Fig. 3C). Similar results were obtained from cultured $P$.

falciparum, where twice the numbers of infected RBCs were bound by human platelets (counted on Giemsa-stained thin smears) (Fig. 3A, iii and iv, and 3D)

and twice the number of infected RBCs were also CD41 positive (Fig. 3E). Therefore, platelets selectively bind infected RBCs.

The presence of dead parasites in the peripheral circulation was investigated by adapting the terminal deoxynucleotidyl transferase-mediated deoxyuridine triphosphate nick end labeling (TUNEL) technique to thin blood smears. TUNEL stains nuclei containing sheared or degraded DNA, an accompaniment of apoptosis or necrosis. As a positive control, all extra-erythrocytic parasites, which commonly occur in late $P$. chabaudi infections, stained positive for TUNEL (fig. S5). In mice with lower parasitemias (day 7), TUNEL staining of intra-erythrocytic parasites was evident (Fig. 
4A), indicative of intraerythrocytic parasite death.

Twice as many dead intra-erythrocytic parasites were seen in C57BL/6 mice as in $\mathrm{Mpl}^{-/-}$mice (Fig. 4C). Although no differences in total parasitemia between $\mathrm{Mpl}^{-/-}$and C57BL/6 were detected at this time point (most TUNEL-stained parasites are morphologically indistinguishable from live parasites), parasitemias measured at later time points were significantly higher in $\mathrm{Mpl}^{-/-}$than in C57BL/6 mice (fig. S6). This suggests that platelet-mediated parasite killing influences parasite numbers past the platelet nadir, resulting in higher parasitemias in $\mathrm{Mpl}^{-/-}$mice, and explains their increased susceptibility.

TUNEL--stained intracellular parasites were also observed in the P. falciparum cultures (Fig. 4B). The frequency of stained parasites was more than three times as high in platelet-treated cultures (Fig. 4D). Significantly higher proportions of TUNEL-stained than non-stained infected RBCs were bound by human platelets (by staining with antibodies to CD41) (Fig. 4E). The greater numbers of dead intra-erythrocytic parasites in the presence of normal numbers of platelet in vivo and in vitro implies that platelets directly kill malarial parasites.

Increased mortality in platelet-deficient mice and direct killing of $P$. falciparum by human platelets indicate that platelets are important in controlling malarial infection. Thrombocytopenia occurs early in infection in both humans and mice, before severe forms of the disease develop $(3,6)$. Therefore, the major protective effect of platelets will be early in infection. Modulation of parasite growth by platelets will buffer the parasite growth rate and allow the engagement of the adaptive immune response. This finding is in addition to the well-established role for platelets in cerebral disease $(15,23,24)$. We have also shown that inhibition of platelet activation abrogates the protective effect, which could explain the deleterious effect aspirin may have on malarial outcome (25). 


\section{References and Notes}

1. G. Min-Oo, P. Gros, Cell. Microbiol. 7, 753 (2005).

2. A. D. Adedapo, C. O. Falade, R. T. Kotila, G. O. Ademowo, J. Vector Borne Dis. 44, 266 (2007).

3. U. Hellgren et al., Bull. World Health Organ. 67, 197 (1989).

4. Z. A. Jeremiah, E. K. Uko, Platelets 18, 469 (2007).

5. A. Kakar, S. Bhoi, V. Prakash, S. Kakar, Diagn. Microbiol. Infect. Dis. 35, 243 (1999).

6. M. D. Oh et al., Am. J. Trop. Med. Hyg. 65, 143 (2001).

7. F. J. DeGraves, H. W. Cox, J. Parasitol. 69, 262 (1983).

8. R. D. Horstmann, M. Dietrich, U. Bienzle, H. Rasche, Blut 42, 157 (1981).

9. S. Ladhani, B. Lowe, A. O. Cole, K. Kowuondo, C. R. Newton, Br. J. Haematol. 119, 839 (2002).

10. P. Gerardin et al., Am. J. Trop. Med. Hyg. 66, 686 (2002).

11. K. Chotivanich et al., J. Infect. Dis. 189, 1052 (2004).

12. A. Pain et al., Proc. Natl. Acad. Sci. U.S.A. 98, 1805 (2001).

13. F. Peyron, B. Polack, D. Lamotte, L. Kolodie, P. Ambroise-Thomas, Parasitology 99, 317 (1989).

14. B. Polack, F. Delolme, F. Peyron, Haemostasis 27, 278 (1997).

15. G. E. Grau et al., J. Infect. Dis. 187, 461 (2003).

16. J. Lou et al., Am. J. Pathol. 151, 1397 (1997).

17. M. R. Yeaman, A. S. Bayer, in Platelets, A. D. Michelson, Ed. (Academic Press, Burlington, MA, 2007), pp. 727-755.

18. W. S. Alexander, A. W. Roberts, N. A. Nicola, R. Li, D. Metcalf, Blood 87, 2162 (1996).

19. H. J. Weiss, L. M. Aledort, S. Kochwa, J. Clin. Invest. 47, 2169 (1968).

20. L. F. Brass, T. J. Stalker, L. Zhu, D. S. Woulfe, in Platelets A. D. Michelson, Ed. (Academic Press, Burlington, MA, 2007), pp. 319-346.

21. S. Rex, J. E. Freedman, in Platelets, A. D. Michelson, Ed. (Academic Press, Burlington, MA, 2007), pp. 251-280.

22. M. Cattaneo, in Platelets A. D. Michelson, Ed. (Academic Press, Burlington, MA, 2007), pp. 201-220.

23. G. E. Grau et al., Eur. Cytokine Netw. 4, 415 (1993).

24. S. C. Wassmer et al., J. Immunol. 176, 1180 (2006).

25. M. English et al., Lancet 347, 1736 (1996). 
26. We thank M. Cozens for flow cytometry support; D. Senyschen, G. Panoschi, and F. Rodda for technical support; the Australian Red Cross Blood Service for providing purified red blood cells; S. Jackson for advice; R. Anders for providing the P. falciparum parasites; and C. Flowers for manuscript preparation. Funding support was from the National Health and Medical Research Council of Australia (Program Grants 490037 and 461219), Australian Cancer Research Foundation, and Howard Hughes Medical Institute. Statistical Analysis. $P$ values were calculated by means of two-tailed $t$ tests, except where specifically mentioned. Ethics approval for animal experiments was received from the Royal Melbourne Hospital, Melbourne, Australia (2002.053), and University of Tasmania (A0008702). Ethics approval for the platelet donations was received from Human Research Ethics Committee (Tasmania) Network (H0009004).

\section{Supporting online material contents}

www.sciencemag.org/cgi/content/full/VOL/ISSUE/PAGE/DC1 Methods

Figs. S1 to S6 References

23 September 2008; accepted 4 December 2008 
Fig. 1. Survival data and parasitological and hematological kinetics of $P$. chabaudi infection in C57BL/6 and $\mathrm{Mpl}^{-/-}$mice. (A and B) Survival curves for $\mathrm{Mpl}^{-/-}$(red) and C57BL/6 (black) mice infected with P. chabaudi and monitored for survival. (C and D) Effect of aspirin or vehicle-only treatment on survival of $\mathrm{Mpl}^{-/-}$and C57BL/6 mice. Given that gender contributes a significant effect, the females (A and C) and males (B and D) have been separated. (E and F) Parasite and hematological parameters in C57BL/6 (E) and $\mathrm{Mpl}^{-/-}$(F) mice. Parasitemias (percentage of infected RBCs), RBC counts $\left(\times 10^{12} /\right.$ liter $)$, and platelet counts $\left(\times 10^{9} /\right.$ liter $)$.

Fig. 2. Platelet-mediated growth inhibition of P. falciparum cultured in human RBCs. (A) Parasite growth inhibition (relative to Tyrode's buffer-treated cultures) with increasing concentrations of purified platelets. Chloro, parasites incubated with $640 \mathrm{nM}$ chloroquine. (B) Parasite growth inhibition with platelets $(50 \mathrm{million} / \mathrm{ml})$ pre-incubated in platelet activation inhibitors at the indicated concentrations. Control, untreated platelets; PGE1, prostaglandin E1; SNP, sodium nitroprusside; MRS2179, $\mathrm{P}_{2} \mathrm{Y}_{1}$ receptor antagonist; MRS2395, P2 $Y_{12}$ receptor antagonist. (C) Inhibition of parasite growth in the presence of platelets $(50 \mathrm{million} / \mathrm{ml})$ pre-incubated with aspirin before addition to culture (in vitro aspirin) or untreated (control) compared with platelets taken from the same donor on separate occasions after treatment with aspirin $(4.2 \mathrm{mg} / \mathrm{kg}$ taken orally twice daily for 1 week before platelet collection) (in vivo aspirin). All experiments were performed with washed platelets. Drugs used alone did not affect parasite growth. Bars for graphs represent the means of at least three independent experiments (A and B) and two independent experiments $(\mathrm{C})$. Error bars indicate \pm 1 SD.

Fig. 3. Infected RBCs are preferentially bound by platelets. (A) Giemsa-stained thin blood smears showing individual RBC-bound platelets (black arrows) [(i) and (ii), mouse; (iii) and (iv), human]. Some parasites look dysmorphic (red arrows) compared with normal parasites (blue arrows) (B) The percentage parasitemia (counted on Giemsastained thin smears) of all mouse RBCs compared to only those with platelets bound ( $P<$ 0.01). (C) The percentage CD41 staining of thiazole orange (TO) positive (infected) versus TO-negative (uninfected) mouse RBCs $(P<0.05)(\mathrm{D})$ Percentage of $P$.

falciparum-infected and uninfected human RBCs bound by platelets counted on Giemsastained thin smears $(P<0.05)$. (E) Percentage of infected (TO- positive) and uninfected (TO-negative) human RBCs that stained positively for CD41 $(P$ $<0.01$ ). (B) and (C) refer to the in vivo mouse model (day 8 of infection) and (D) and (E) to the in vitro human falciparum culture model (after 24 hours of incubation with platelets). Bars for graphs represent mean of three independent experiments. Error bars represent $\pm 1 \mathrm{SD}$.

Fig. 4. Evidence that intra-erythrocytic parasites are killed by platelets. (A) TUNEL staining of a $P$. chabaudi-infected C57BL/6 thin blood smear (day 7 of infection). (i) Hoechst staining of parasite DNA. (ii) TUNEL staining of parasite DNA, and (iii) both TUNEL and Hoechst staining superimposed on dark-field microscopy showing intraerythrocytic parasites. (B) Immunofluorescent images of $P$. falciparum-parasitized human RBCs cocultured with platelets for 24 hours. Separate images of cells stained 
with (i), Hoechst -(all parasites), (ii) anti-CD41 -(platelets), and (iii) TUNEL - (dead parasites); (iv) merged image of (i), (ii), and (iii) (anti-CD41, red; TUNEL, green; Hoechst, blue). (C) The percentage of all $P$. chabaudi parasites staining positive with TUNEL in C57BL/6 and $\mathrm{Mpl}^{-/-}$mice $(P<0.05)$. (D) Percentage of TUNEL- positive parasites in $P$. falciparum cultures incubated with platelets $(50$ million $/ \mathrm{ml})$ and without platelets for 24-hours $(P<0.05)$. (E) Percentage of $P$. falciparum-parasitized RBCs bound by platelets that stained positively or negatively with TUNEL stain $(P<0.05)$. Bars for graphs represent the mean of at least three independent experiments with error bars representing $\pm 1 \mathrm{SD}$. 
A

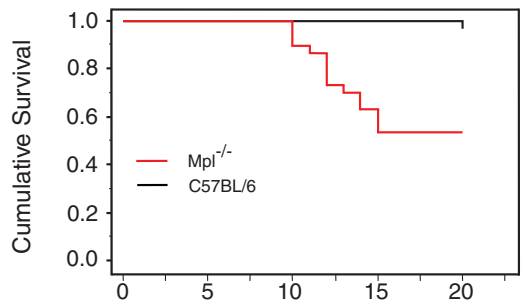

C

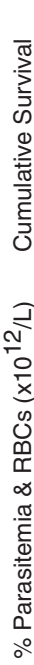

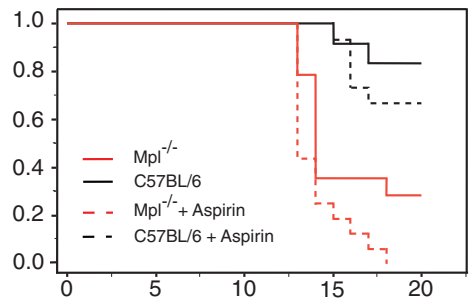

\section{$E$}

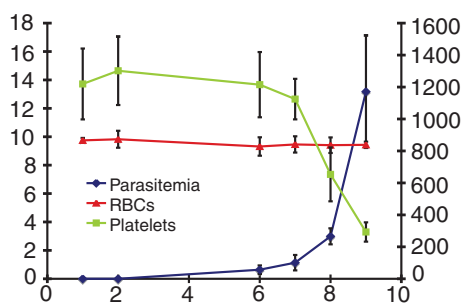

Days post inoculation

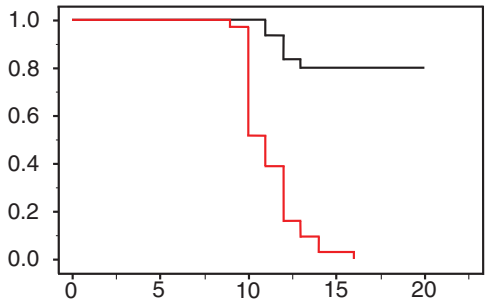

D

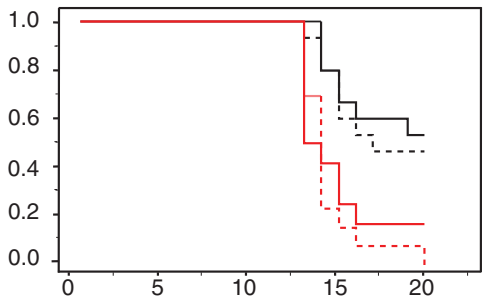

F

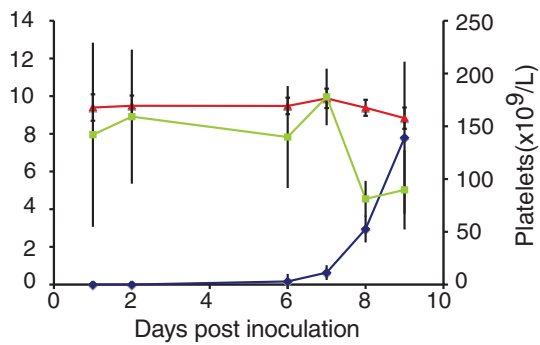



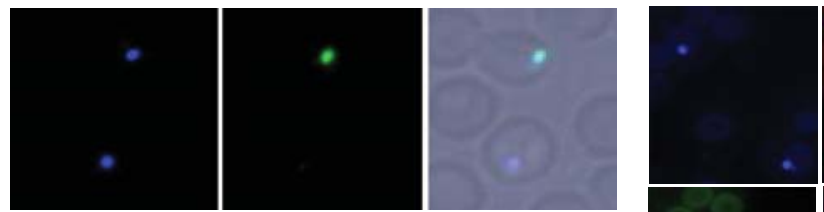

D

C
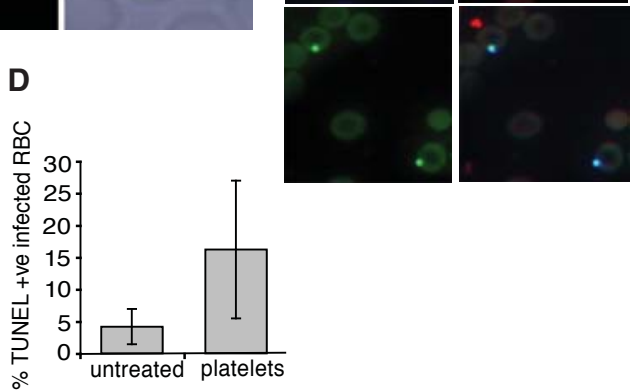

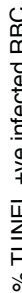

E

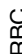

(

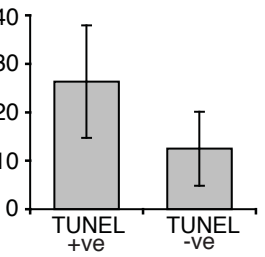

